\title{
A pilot study to evaluate the role of peripheral blood CD4+CRTh2+CCR6+ in predicting future asthma in preschool wheezing children: Outcome of two-year follow-up
}

\author{
Jingyang $\mathrm{Li}^{1}$, Jinhong $\mathrm{Wu}^{2}$, Haipei $\mathrm{Liu}^{1}$, Li Hua ${ }^{1}$, Quanhua Liu ${ }^{1}$, Ruoxu $\mathrm{Ji}^{1}$, Dingzhu \\ Fang $^{1}$, Yi Chen ${ }^{1}$, Jianhua Zhang ${ }^{1}$, and wenwei zhong ${ }^{1}$ \\ ${ }^{1}$ Xinhua Hospital Affiliated to Shanghai Jiaotong University School of Medicine \\ ${ }^{2}$ Shanghai Childrens Medical Center Affiliated to Shanghai Jiaotong University School of \\ Medicine
}

July 16, 2020

\begin{abstract}
Background:Wheezing is an important respiratory symptom in the diagnosis of asthma. However, wheezing is common in children and often related to viral infection. This and the lack of reliable biological indicators for asthma create difficulty in diagnosing asthma early in children. Objective: In this study, the levels of CD4+CCR6+CRTh2+ memory Th2 cells in wheezing children with different diagnostic outcomes were investigated to determine correlation with a diagnosis of asthma and to assess their potential clinical value as a biological indicator for this disease. Methods: A prospective study was performed with a cohort of wheezing children aged 3 months to 6 years hospitalized in the Division of Pulmonary Pediatrics at Shanghai Xinhua hospital and Shanghai children's medical center affiliated to Shanghai Jiao Tong University School of Medicine from July 2017 to March 2018. After inclusion, the level of serum IgE, presence of allergen-specific serum IgE (sIgE) and proportion of circulating CD4+CCR6+CRTh2+ memory Th2 cells were counted. In addition, the patients' personal and family histories of allergic disease were acquired by questionnaire. The children were followed up annually over 2 years by telephone call with a guardian to record whether the child had been diagnosed with asthma. The accuracy of an increased proportion of CD4+CCR6+CRTh2+ memory Th2 cells as an indicator of asthma was assessed. Results: A total of 43 children completed follow-up. The median of circulating CD4+CCR6+CRTh2+ memory Th2 cells in wheezing children diagnosed with or without asthma was $1.2 \%(0.8 \% \sim 2.9 \%)$ and $0.6 \%(0.1 \% \sim 0.9 \%)$, respectively, and was significantly higher in children diagnosed with asthma $(\mathrm{P}<0.01)$. The median of circulating CD4+CCR6+CRTh2+ memory Th2 cells in atopic children was also significantly higher in children diagnosed with asthma than in children without asthma, at $1.3 \%\left(0.8 \%^{\sim} 2.9 \%\right)$ and $0.6 \%\left(0.3 \%^{\sim} 1.0 \%\right)$, respectively, $(\mathrm{P}<0.01)$. Furthermore, the level of serum $\operatorname{IgE}$ was significantly higher in children with asthma $(\mathrm{P}<0.05)$. Logistic regression analysis indicated that the level of circulating CD4+CCR6+CRTh2+ memory Th2 cells were independent risk factors for asthma. The area under the receiver operating characteristic curve (ROC) was 0.922 . There was no significant difference in the positive rate of memory Th2 cells in the context of allergic rhinitis (AR) or atopic dermatitis (AD) $(\mathrm{P}>0.05)$. Conclusion: Our exploratory study found that an increase in the level of circulating CD4+CCR6+CRTh2+ memory Th2 cells could be used as a biological indicator for early diagnosis of asthma, especially in predicting the risk of asthma in atopic children.
\end{abstract}

\section{Introduction}

Asthma is a common chronic inflammatory disease of the airway in children. The most typical symptom of asthma is recurrent wheezing. However, wheezing is common in preschool children which is commonly related to acute respiratory tract infection. The Modified Asthma Predictive Index (mAPI) is a practical method commonly used in the clinic; however, its sensitivity and specificity are limited. Therefore, it is of 
great clinical value to explore biological indicators based on the immunology of bronchial asthma to guide the diagnosis and treatment of asthma more effectively.

Pathogenic memory type Th2 cells play an important role in allergic airway inflammation. CD4 ${ }^{+}$ memory $\mathrm{T}$ cells can aggravate allergic airway inflammation by inducing eosinophilic aggregation, mucus hypersecretion $^{(1)}$ and airway hyperresponsiveness ${ }^{(2)}$. Conversely, inhibition of memory Th2 cells can alleviate allergic airway inflammation and improve the symptoms of asthma ${ }^{(3)}$. These studies suggest that memory Th2 cells reflect previous allergic inflammation and can distinguish wheezing caused by non-allergic inflammation versus an immunological mechanism.

In this study, a cohort of wheezing children was established. The patient and family histories of allergic diseases were recorded in detail. In addition, the proportion of circulating CD $4{ }^{+} \mathrm{CCR} 6{ }^{+} \mathrm{CRTh} 2^{+}$memory $\mathrm{Th} 2$ cells were measured, and the wheezing children were followed up for 2 years to confirm whether they were diagnosed with asthma. Statistical methods were used to determine the correlation between the level of circulating $\mathrm{CD} 4^{+} \mathrm{CCR} 6^{+} \mathrm{CRTh} 2^{+}$memory $\mathrm{Th} 2$ cells and a diagnosis of asthma, thereby providing a theoretical basis for the early diagnosis of asthma in children.

\section{Methods}

\section{Study design and subjects}

From July 2017 to March 2018, wheezing Chinese children aged 3 months to 6 years hospitalized or in clinic in the Division of Pulmonary Pediatrics at Shanghai Xinhua hospital and Shanghai children's Medical center affiliated to Shanghai Jiao Tong University School of Medicine were enrolled in this study (wheezing was confirmed by doctors at the time of admission or from documentation in medical records). Exclusion criteria were: 1) bronchopulmonary dysplasia, tracheobronchial foreign bodies or congenital heart disease; 2) acute and chronic infectious diseases; 3) immunomodulatory treatment before or during the study; and 4) the researchers considered the patient inappropriate for inclusion. The study was examined and approved by the Ethics Committee of Shanghai Xinhua hospital Affiliated to Shanghai Jiao Tong University School of Medicine and the guardians of all included children provided signed informed consent. The history of allergic diseases (AR and AD diagnosed by specialists) and family allergic history were collected through questionnaires filled out by the parents. Additionally, respiratory symptoms and history of anti-allergy drug use were provided by the family members and recorded for the study. At inclusion, $2 \mathrm{ml}$ peripheral blood was taken without anticoagulant from all the patients for allergen-specific IgE testing, and $1 \mathrm{ml}$ blood using heparin as anticoagulant was collected to determine the proportion of circulating $\mathrm{CD} 4^{+} \mathrm{CCR} 6{ }^{+} \mathrm{CRTh} 2^{+}$memory-type Th2 cells flow-cytometrically.

\section{Laboratory tests}

\section{Measurement of serum allergen-specific sIgE}

An allergen-specific sIgE test was conducted on blood from all children included in the study using a DX-Blot 45 Automatic Western Blotting instrument (Hangzhou Zheda Dixun Biological Gene engineering Company, Zhejiang, China) according to the manufacturer's instructions.

\section{Detection of $\mathrm{CD}^{+}{ }^{+\mathrm{CCR}}{ }^{+} \mathrm{CRTH}^{+}{ }^{+}$memory $\mathrm{Th} 2$ cells}

Blood $(100 \mu \mathrm{l})$, with heparin as anticoagulant, was mixed with PE-labeled anti-CRTH2, PE-Cy5.5-labeled anti-CD4 and PE-Cy7-labeled anti-CCR6 monoclonal antibodies and incubated at room temperature avoiding light for 30 minutes. Red blood cells were removed by lysis, and CD4 ${ }^{+} \mathrm{CCR} 6{ }^{+} \mathrm{CRTH} 2^{+}$memory Th2 cells were detected by CytoFLEX flow cytometry. Data analysis was performed using CytExpert version 2.3.0.84.

\section{Follow up}

The included children were followed up for 2 years via an annual telephone interview or in clinic. The frequency of $\mathrm{AR}, \mathrm{AD}$, wheezing episodes, and asthma diagnosis were recorded. Asthma was diagnosed by 
pediatric respiratory specialists according to the Global Initiative for Asthma (GINA) standard.

\section{Statistical analysis}

All statistical analysis was carried out using SPSS v. 22.0 . Continuous variables were represented by median (interquartile range, IQR). Classification variables were represented by frequency. Mann-Whitney rank sum test was used to compare the non-normally distributed data. Logistic regression was used for correlation analysis. The area under the curve was calculated by the ROC of the subjects. Bilateral significance level was set at $\mathrm{P}<0.05$.

\section{Results}

\section{Subject characteristics and demographics}

A total of 45 children were enrolled in this study. Among them, 43 cases (95.6\%) underwent blood tests and completed follow-up (Figure 1). Nineteen $(44.2 \%)$ of the included patients were girls, and the age distribution was as follows: 15 cases were aged 3 months to 1 year (34.9\%), and 28 cases were $1-5$ years old (65.1\%). A total of $79.1 \%$ of the subjects had other allergic diseases, including AD (62.8\%) and AR (51.2\%). Twenty-six $(60.5 \%)$ of the children had a family history of allergies. Characteristics of the patients are listed in table 1.

\section{Atopic feature in wheezing children with or without asthma diagnosis}

There was no significant difference in the incidence of AD and AR between the asthma $(66.7 \%$ and $51.6 \%$, respectively) and the non-asthma (58.3\% and $50 \%$, respectively) groups. There was also no significant difference in family history of $\mathrm{AR}, \mathrm{AD}$ or asthma between the asthma (48.3\%, $10.7 \%$ and $32.1 \%$, respectively) and the non-asthma $(25.0 \%, 8.3 \%$ and $25.0 \%$, respectively) groups(Table 2$)$.

\section{Serum allergen-specific IgE in wheezing children diagnosed with asthma or without asthma.}

The presence of allergen-specific sIgE was assessed in 43 subjects. Thirty-two cases $(74.4 \%)$ were positive for antigen-specific sIgE; 23 cases (53.5\%) had sIgE specific for inhaled allergens and 9 cases (20.9\%) specific for food allergens. Eleven subjects were negative for all allergen-specific antibodies tested (table 1).

We found the positive rates of inhaled-allergen-specific sIgE and arbitrary sIgE (including inhaled allergen and food allergen) in asthma group were $63.3 \%$ and $76.7 \%$, respectively, while the positive rates in nonasthma group were $33.3 \%$ and $75.0 \%$, respectively. There was no significant difference in the presence of inhaled-allergen-specific sIgE $(\mathrm{P}>0.05)$ or arbitrary-allergen-specific $\operatorname{sIgE}(\mathrm{P}>0.05)$ between the two groups (Table 2).

$\mathrm{CD}^{+}{ }^{\mathrm{CCR}^{+}}{ }^{+} \mathrm{CRTH2}^{+}$Memory Th2 cells are associated with asthma but not with AD or AR, especially in atopic children

To investigate whether memory Th2 cells can distinguish children with or without asthma, $\mathrm{CD} 4{ }^{+} \mathrm{CCR} 6{ }^{+} \mathrm{CRTH} 2^{+}$memory Th 2 cells were measured in all children at time of inclusion and the results were used to correlate with diagnosis after the 2-year follow-up. In wheezing children diagnosed with asthma, the level of peripheral blood $\mathrm{CD} 4^{+} \mathrm{CCR} 6^{+} \mathrm{CRTH} 2^{+}$was $1.2 \%(0.8 \% \sim 2.9 \%)$, while the level in children without asthma was $0.6 \%(0.1 \% \sim 0.9 \%)$. There was a significant difference in the level of $\mathrm{CD} 4^{+} \mathrm{CCR} 6{ }^{+} \mathrm{CRTH} 2^{+}$memory Th2 cells at inclusion in children with asthma compared with the non-asthma group $(\mathrm{P}<0.01)$ (Table 3 ). In atopic children(any history of $\mathrm{AD}$ or $\mathrm{AR}$ ), the level of peripheral blood $\mathrm{CD} 4^{+} \mathrm{CCR} 6^{+} \mathrm{CRTH} 2^{+}$was $1.3 \%(0.8 \% \sim 2.9 \%)$, while the level in children without asthma was $0.6 \%\left(0.3 \%^{\sim} 1.0 \%\right)$. There was a significant difference in the level of $\mathrm{CD} 4{ }^{+} \mathrm{CCR} 6{ }^{+} \mathrm{CRTH} 2^{+}$memory $\mathrm{Th} 2$ cells at inclusion in children with asthma compared with the non-asthma group $(\mathrm{P}<0.01)$ (Table 3$)$.

Because asthma is primarily a pulmonary manifestation of systemic allergic reaction, we further explored the relationship between the level of $\mathrm{CD} 4^{+} \mathrm{CCR} 6{ }^{+} \mathrm{CRTh} 2^{+}$memory Th2 cells and allergic diseases such as $\mathrm{AR}$ and AD. There was no significant difference in the level of $\mathrm{CD} 4{ }^{+} \mathrm{CCR} 6{ }^{+} \mathrm{CRTh} 2{ }^{+}$cells between the AR group and the non-AR group $(\mathrm{P}>0.05)$, as well as between the $\mathrm{AD}$ group and the non-AD group $(\mathrm{P}>0.05$, Table 3$)$. 
The level of CD4 ${ }^{+} \mathrm{CCR}^{+}{ }^{+} \mathrm{CRTh2}{ }^{+}$memory $\mathrm{Th} 2$ cells is an effective index for the diagnosis of asthma in wheezing children.

There were significant differences in levels of serum IgE, level of CD4 ${ }^{+} \mathrm{CCR} 66^{+} \mathrm{CRTh} 2^{+}$memory Th2 cells and age between children diagnosed with asthma and children with non-asthma outcomes. Binary logistic regression analysis and the Wald $\mathrm{X}^{2}$ test of each regression coefficient showed that the level of memory-type Th2 cells (OR, 29.926; 95\% CI, 1.791-499.933; $\mathrm{P}=0.018)$ was independent risk factors for asthma in these children (Table 4). The ROC was adopted to calculate the sensitivity and specificity of the model. The area under the ROC curve was 0.922 (95\%CI 0.836-1.000; $\mathrm{P}<0$. 001.Figure 2).

\section{Discussion}

Asthma is generally diagnosed based on the clinical manifestations of recurrent wheezing combined with reversible airflow obstruction. Unlike for adults, there are no objective criteria for the diagnosis of asthma in children under 6 years of age, and especially for those under 3 years of age. At present, the diagnosis and treatment of asthma in this age group is mainly based on the frequency of wheezing attacks and the prediction of high asthma risk based on the $\mathrm{mAPI}^{(4,5)}$. However, the positive rate of asthma prediction based on mAPI is limited ${ }^{(6,7)}$. Therefore, although atopy is a high-risk factor for asthma, many these children will not develop asthma. There is an urgent need for stable and specific biological indicators to assist in the diagnosis of asthma, and a major focus of research in the field is to find specific biological indicators through the immunological mechanism of asthma ${ }^{(8,9)}$.

In this study, a small-sample cohort of 43 wheezing children was established. The relationship between the level of circulating $\mathrm{CD} 4{ }^{+} \mathrm{CCR} 6{ }^{+} \mathrm{CRTh} 2^{+}$memory $\mathrm{Th} 2$ cells and a diagnosis of asthma was determined. We found that the level of $\mathrm{CD} 4^{+} \mathrm{CCR} 6^{+} \mathrm{CRTh} 2^{+}$memory Th2 cells in the asthma group was significantly higher than that in the non-asthma group. Logistic regression analysis further indicated that $\mathrm{CD} 4{ }^{+} \mathrm{CCR} 6{ }^{+} \mathrm{CRTh} 2^{+}$memory $\mathrm{Th} 2$ cells were an independent risk factor for the diagnosis of asthma. In this study, $74.4 \%$ of the subjects showed inhaled and/or food allergen sensitization, indicating that most of the included patients had allergic physique. Therefore, we analyzed whether there were differences in $\mathrm{CD} 4^{+} \mathrm{CCR} 6{ }^{+} \mathrm{CRTh} 2^{+}$memory Th2 cells among other allergic diseases. The increase in $\mathrm{CD} 4^{+} \mathrm{CCR} 6^{+} \mathrm{CRTh} 2^{+}$memory Th2 cells is specifically related to asthma and is not affected by AR or AD. $\mathrm{CD} 4^{+} \mathrm{CCR} 6{ }^{+} \mathrm{CRTh} 2^{+}$memory Th2 cells have certain tissue specificity and reflect allergic airway inflammation. This result can be further demonstrated by the fact that higher level of CD $4^{+} \mathrm{CCR} 6{ }^{+} \mathrm{CRTh} 2^{+}$memory Th2 cells in asthmatic children than non-asthmatic children in atopic population.

$\mathrm{CD} 4{ }^{+} \mathrm{CCR} 6{ }^{+} \mathrm{CRTh} 2^{+}$memory Th2 cell is a type of pathogenic memory-type Th2 cell. Pathogenic memorytype Th2 cells are mainly distributed in local tissues for long periods of time and play key roles in the maintenance of chronic allergic inflammation ${ }^{(10-12)}$. CD4 ${ }^{+} \mathrm{CRTH} 2^{+} \mathrm{CCR} 6^{+}$cells are memory Th2 cells that produce IL-17, which is mainly found in the airway. $\mathrm{CD} 4{ }^{+} \mathrm{CRTH} 2^{+} \mathrm{CCR} 6{ }^{+}$cells secrete IL-17, IL-4, and IL-13, leading to the infiltration neutrophils and eosinophils, which is responsible for chronic inflammation associated with asthma. This course of events reflects the immune memory of allergic airway inflammation in the lung tissue. A small number of pathogenic memory-type Th2 cells can enter the circulation ${ }^{(10,11)}$. Therefore, the detection of circulating $\mathrm{CD} 4{ }^{+} \mathrm{CRTH} 2^{+} \mathrm{CCR} 6{ }^{+}$memory Th2 cells producing IL- 17 is an indicator of previous and chronic airway allergic inflammation, an important immunological feature of asthma.

In conclusion, despite of small sample, our exploratory study found that because $\mathrm{CD} 4{ }^{+} \mathrm{CCR} 6{ }^{+} \mathrm{CRTh} 2^{+}$memory Th2 cells have certain lung tissue specificity and an increase in circulating memory Th2 cells reflects previous and present allergic chronic inflammation, these cells can be used as a potential biological indicator for diagnosis of asthma in preschool children, especially in atopic children. We will further expand the sample size and prolong the follow-up time to validate the current results.

\section{Acknowledgments}

We thank Michelle Ryndak, PhD, from Liwen Bianji, Edanz Editing China (www.liwenbianji.cn/ac), for editing the English text of a draft of this manuscript. 


\section{Authors' contributions}

Wenwei Zhong, Jianhua Zhang, Jinyang Li designed the study, followed up patients, analyzed the data, wrote the manuscript, and provided approval for the final version of the manuscript. Haipei Liu, Li Hua, Quanhua Liu, Dingzhu Fang, Yi chen, Ruoxu Ji conducted the experiments, followed up patients and interpreted the data.

\section{Fund}

This work was supported by grants from the Natural Science Foundation of Shanghai (grant number: 17ZR1418000, 12ZR1419100) and the National Natural Science Foundation of China (81270085)

\section{Consent for publication}

Not applicable.

\section{Disclosure of Conflicts of Interest}

The authors declare no competing financial interests

\section{References}

1. Nials AT, Uddin S. Mouse models of allergic asthma: acute and chronic allergen challenge. Dis Model Mech. 2008;1(4-5):213-20.

2. Nakagome K, Dohi M, Okunishi K, To Y, Sato A, Komagata Y, et al. Antigen-sensitized CD4+CD62Llow memory/effector $\mathrm{T}$ helper 2 cells can induce airway hyperresponsiveness in an antigen free setting. Respir Res. 2005;6:46.

3. Huang CH, Loo EX, Kuo IC, Soh GH, Goh DL, Lee BW, et al. Airway inflammation and IgE production induced by dust mite allergen-specific memory/effector Th2 cell line can be effectively attenuated by IL-35. J Immunol. 2011;187(1):462-71.

4. Boulet LP, Reddel HK, Bateman E, Pedersen S, FitzGerald JM, O'Byrne PM. The Global Initiative for Asthma (GINA): 25 years later. Eur Respir J. 2019;54(2).

5. Serebrisky D, Wiznia A. Pediatric Asthma: A Global Epidemic. Ann Glob Health. 2019;85(1).

6. Castro-Rodriguez JA. The Asthma Predictive Index: early diagnosis of asthma. Curr Opin Allergy Clin Immunol. 2011;11(3):157-61.

7. Wi CI, Krusemark EA, Voge G, Sohn S, Liu H, Ryu E, et al. Usefulness of asthma predictive index in ascertaining asthma status of children using medical records: An explorative study. Allergy. 2018;73(6):127683.

8. Gans MD, Gavrilova T. Understanding the immunology of asthma: Pathophysiology, biomarkers, and treatments for asthma endotypes. Paediatr Respir Rev. 2019.

9. McDowell PJ, Heaney LG. Different endotypes and phenotypes drive the heterogeneity in severe asthma. Allergy. 2020;75(2):302-10.

10. Endo Y, Hirahara K, Yagi R, Tumes DJ, Nakayama T. Pathogenic memory type Th2 cells in allergic inflammation. Trends Immunol. 2014;35(2):69-78.

11. Endo Y, Nakayama T. PATHOGENIC MEMORY Th2 CELLS AND ALLERGIC DISEASES. Arerugi. 2016;65(9):1182-6.

12. Hirahara K, Shinoda K, Endo Y, Ichikawa T, Nakayama T. Maintenance of memory-type pathogenic Th2 cells in the pathophysiology of chronic airway inflammation. Inflamm Regen. 2018;38:10.

\section{Hosted file}


Table.docx available at https://authorea.com/users/342182/articles/469041-a-pilot-studyto-evaluate-the-role-of-peripheral-blood-cd4-crth2-ccr6-in-predicting-future-asthma-inpreschool-wheezing-children-outcome-of-two-year-follow-up

\section{Hosted file}

Figure 1.docx available at https://authorea.com/users/342182/articles/469041-a-pilot-studyto-evaluate-the-role-of-peripheral-blood-cd4-crth2-ccr6-in-predicting-future-asthma-inpreschool-wheezing-children-outcome-of-two-year-follow-up

\section{Hosted file}

Figure 2.docx available at https://authorea.com/users/342182/articles/469041-a-pilot-studyto-evaluate-the-role-of-peripheral-blood-cd4-crth2-ccr6-in-predicting-future-asthma-inpreschool-wheezing-children-outcome-of-two-year-follow-up 